\title{
Magnetic tension of sunspot fine structures ${ }^{\star}$
}

\author{
P. Venkatakrishnan and Sanjiv Kumar Tiwari
}

Udaipur Solar Observatory, Physical Research Laboratory, Dewali, Bari Road, Udaipur-313 001, India
e-mail: [pvk; stiwari] @prl.res.in

Received 14 April 2010 / Accepted 20 May 2010

\section{ABSTRACT}

\begin{abstract}
Context. The equilibrium structure of sunspots depends critically on its magnetic topology and is dominated by magnetic forces. Tension force is one component of the Lorentz force, which balances the gradient of magnetic pressure in force-free configurations. Aims. We employ the tension term of the Lorentz force to clarify the structure of sunspot features like penumbral filaments, umbral light bridges, and outer penumbral fine structures.

Methods. We computed the vertical component of the tension term of Lorentz force over two active regions, NOAA AR 10933 and NOAA AR 10930 observed on 5 January 2007 and 12 December 2006, respectively. The former is a simple active region while the latter is a complex one with highly sheared polarity inversion line (PIL). We obtained the vector magnetograms from Hinode(SOT/SP). Results. We find an inhomogeneous distribution of tension with both positive and negative signs in various features of the sunspots. The existence of positive tension at locations of lower field strength and higher inclination is compatible with the uncombed model of the penumbral structure. Positive tension is also seen in umbral light bridges, which could be indication of uncombed structure of the light bridge. Likewise, the upwardly directed tension associated with bipolar regions in the penumbra could be a direct confirmation of the sea serpent model of penumbral structures. Upwardly directed tension at the PIL of AR 10930 seems to be related to flux emergence. The magnitude of the tension force is greater than the force of gravity in some places, implying a nearly force-free configuration for these sunspot features.
\end{abstract}

Conclusions. From our study, magnetic tension emerges as a useful diagnostic of the local equilibrium of the sunspot fine structures.

Key words. Sun: photosphere - sunspots - magnetic fields

\section{Introduction}

The equilibrium structure of sunspots is obviously dominated by magnetic forces. Early research on this problem dealt mainly with the problem of the global equilibrium of sunspots (Meyer et al. 1977). The sunspot was modeled as a magnetic flux rope where the lateral force balance was envisaged as a pressure balance between the photospheric plasma pressure and the magnetic + plasma pressure inside the flux rope (e.g., Chitre 1963). Since the magnetic field is divergence free, the field lines of the flux rope must bend back into the photosphere resulting in a "closed" field topology. The resulting curvature of the field lines produces a magnetic tension that should basically have a downward vertical component. The model of Meyer et al. (1977) also put constraints on the field line curvature, since the equilibrium becomes unstable when the radius of curvature is shorter than a certain value. With the availability of high-resolution magnetograms of sunspots, it has become clear that the earlier models of sunspots might no longer be adequate to explain the dynamical equilibrium of various fine structures seen in the umbra, as well as in the penumbra.

One simple diagnostic of the vertical equilibrium is the vertical component of the magnetic tension, which can be determined from the lateral gradients of the vector magnetic field. Information about the vertical component of the magnetic tension has already been found to be very useful, as seen in the correlation of low tension force with large magnetic shear in early vector magnetograms (Venkatakrishnan et al. 1993) measured by the MSFC vector magnetograph (Hagyard et al. 1982).

^ Figures A.1-A.3 are only available in electronic form at http: //www . aanda. org
The magnetic tension measurements at the polarity inversion lines underlying filaments/prominences are likewise very important, since the vanishing of magnetic tension at these highly sheared locations makes the prominence structure extremely vulnerable to dynamical instabilities, via thermal instabilities (Venkatakrishnan 1990b).

Modern observations of sunspots have revealed the existence of different fine structures. In the umbra we have the umbral dots and light bridges (Sobotka 1989; Sobotka et al. 1997b,a). In the penumbra we have spines (stronger, more vertical field) wrapping around the intraspines (weaker, more horizontal field) (Lites et al. 1993; Borrero et al. 2008). A few models have also been proposed to explain these structures such as uncombed model of Solanki \& Montavon (1993) or the gappy model of Spruit \& Scharmer (2006).

In this paper, we investigate the height variation of the sunspot fine structure using the calculations of the vertical component of the magnetic tension force. The expression for computing the vertical component of tension force is given in Sect. 2. In Sect. 3, we describe the data sets used. Section 4 describes the analysis and results. Finally in Sect. 5 we present our conclusions.

\section{Brief description of the magnetic tension force}

In any plasma with magnetic field $\boldsymbol{B}$ and plasma pressure $p$, the equation for magneto-hydrostatic equilibrium is given by (Parker 1979)

$(\nabla \times \boldsymbol{B}) \times \boldsymbol{B} / 4 \pi-\nabla p+\rho \boldsymbol{g}=0$ 
where $\rho$ is the plasma density and $\boldsymbol{g}$ the acceleration due to gravity. The first term in Eq. (1) is the Lorentz force, the second term is the force due to plasma pressure, and the last term is the force on the plasma due to gravity. We can split up the Lorentz force (say $\boldsymbol{F}$ ) in two terms as

$\boldsymbol{F}=\frac{(\boldsymbol{B} \cdot \nabla) \boldsymbol{B}}{4 \pi}-\frac{\nabla(\boldsymbol{B} \cdot \boldsymbol{B})}{8 \pi}$.

The first term in this equation is the tension force (say $\boldsymbol{T}$ ). The second term represents the force due to magnetic pressure. The vertical component of the tension term can be expanded in terms of the horizontal derivatives of the magnetic field as

$T_{z}=\frac{1}{4 \pi}\left[B_{x} \frac{\partial B_{z}}{\partial x}+B_{y} \frac{\partial B_{z}}{\partial y}-B_{z}\left(\frac{\partial B_{x}}{\partial x}+\frac{\partial B_{y}}{\partial y}\right)\right]$

where the last component is drawn from the condition

$\nabla \cdot \boldsymbol{B}=0$.

The utility of the tension force as a diagnostic of dynamical equilibrium has not had much attention in the literature so far except in a restricted sense (Venkatakrishnan 1990a,b; Venkatakrishnan et al. 1993). We computed the tension force using Eq. (3) and expressed it in the units of dynes $/ \mathrm{cm}^{3}$.

\section{Data sets used}

We used the vector magnetograms of NOAA AR 10933 observed on 5 January 2007 and NOAA AR 10930 observed on 12 December 2006. These data sets are obtained from the Solar Optical Telescope/Spectro-polarimeter (SOT/SP: Tsuneta et al. 2008; Suematsu et al. 2008; Ichimoto et al. 2008; Shimizu et al. 2008) onboard Hinode (Kosugi et al. 2007).

The Hinode (SOT/SP) data were calibrated by the standard "SP_PREP" routine developed by B. Lites and available in the Solar-Soft package. The "SP_PREP" determines the thermal shifts in the spectral and slit dimensions and also applies the drift corrections for calibrating the data from level0 to level1. The prepared polarization spectra were then inverted to obtain vector magnetic field components using an UnnoRachkowsky (Unno 1956; Rachkowsky 1967) inversion under the assumption of Milne-Eddington (ME) atmosphere (Landolfi \& Landi Degl'Innocenti 1982; Skumanich \& Lites 1987). We used the "STOKESFIT" inversion code ${ }^{1}$, which is available in the Solar-Soft package. The latest version of the inversion code is used, which returns the intrinsic field strengths along with the filling factor. The effect of polarimetric noise on the estimation of vector fields is almost negligible (Tiwari et al. 2009a; Gosain et al. 2010). The $180^{\circ}$ azimuthal ambiguity in our data sets were removed by using the acute angle method (Harvey 1969; Sakurai et al. 1985; Cuperman et al. 1992). The data sets used have a spatial sampling of $\sim 0.32$ arcsec/pixel observed in the "fast mode" scans of SOT/SP. A simple (NOAA AR 10933) and a complex (NOAA AR 10930) sunspot we analyzed. The noise in the data was minimized in a similar way to Tiwari (2009); Tiwari et al. (2009b) and Venkatakrishnan \& Tiwari (2009).

\section{Data analysis and results}

We calculated the vertical component of the tension force for all locations in the sunspots. Figure 1 shows a continuum picture

1 The code has been developed by T. R. Metcalf.

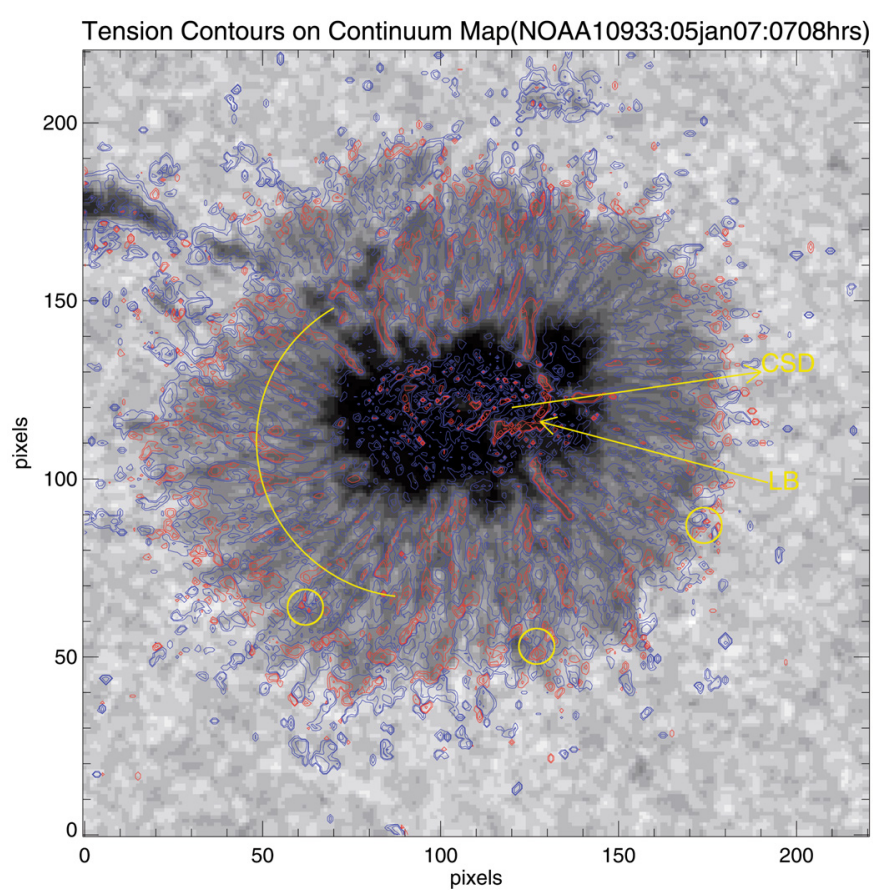

Fig. 1. Contours of tension forces overlaid on the continuum map of NOAA AR 10933 (S04E05). Blue (red) colors show negative (positive) contours of $\pm 1.2, \pm 4, \pm 12$ millidynes $/ \mathrm{cm}^{3}$. The position of a light bridge(LB) and three examples of bipolar sea serpent regions are shown by an arrow and by circles, respectively. An arc is shown for which the scatter plots of tension, field strength, and inclination are shown in Fig. 2. The heliocentric angle is $\theta=8^{\circ}$, and an arrow points towards the center of the solar disk (CSD).

of the NOAA AR 10933 overlaid by the contours of the magnetic tension expressed in units of dynes $/ \mathrm{cm}^{3}$. Several interesting points can be noted. The most obvious result is the existence of locations of positive tension, contrary to the expectation from simple sunspot models having a "closed" field topology. Of further interest is that these locations of positive tension lie along linear structures in the penumbra, which resemble the penumbral filaments. The magnitude of the tension is very comparable to the force of gravity and exceeds the solar gravity in some places. For a more detailed comparison, we show in Fig. A.1 (online), a map of the magnetic field strength overlaid with the contours of vertical tension. In Fig. A.2 (online), we present a map of the inclination overlaid with contours of the magnetic tension. Here again, the locations of positive tension largely coincide with regions of lower magnetic field strength and greater magnetic inclination. In Fig. 2, we show scatter plots of field strength versus tension, as well as inclination versus tension. These scatter plots show a clear trend toward the positive tension being associated with weaker and more inclined fields.

Apart from the penumbral features, we can also see several umbral features, including a light bridge at the western portion of the umbra. Even in this case, the regions of positive tension coincide with locations of weaker and more inclined fields. Finally, we notice several locations of upward directed tension at the edges of the filamentary penumbral structures. In Fig. 3, we show the contours of tension overlaid on the continuum map of NOAA AR 10930. Apart from the similar structures of positive and negative tensions as seen in the NOAA AR 10933 (Fig. 1), the polarity inversion line of NOAA AR 10930 also shows high magnitudes of tension $\left(10^{-2}\right.$ dynes $\left./ \mathrm{cm}^{3}\right)$. 


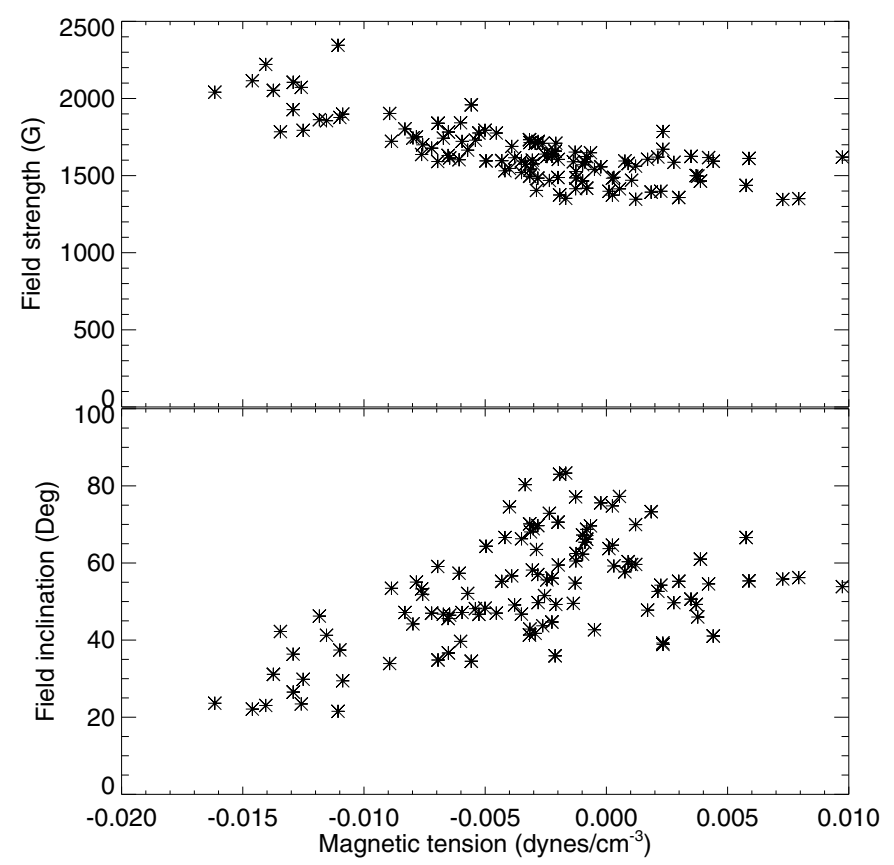

Fig. 2. Upper panel: the scatter plot between magnetic tension and field strength along the arc shown in Fig. 1. Lower panel: the scatter plot between magnetic tension and field inclination along the arc shown in Fig. 1.

\section{Discussion and conclusions}

The variation in magnetic field parameters with optical depth can be obtained (Collados et al. 1994; Westendorp Plaza et al. 2001b,a; Beck 2008) using inversion schemes like SIR (Ruiz Cobo \& del Toro Iniesta 1992, 1994; Del Toro Iniesta \& Ruiz Cobo 1996; del Toro Iniesta 2003). With the availability of high-quality stokes profiles from the Solar Optical Telescope (Tsuneta et al. 2008; Suematsu et al. 2008; Ichimoto et al. 2008; Shimizu et al. 2008) aboard Hinode (Kosugi et al. 2007), these inversions are now possible with unprecedented quality. In particular, this was used to demonstrate the variation of the magnetic field of penumbral filaments as a function of the optical depth (Borrero et al. 2008), which showed the wrapping of the spine around the intraspine. An inspection of Fig. 2 of Borrero et al. (2008) shows that the magnetic field lines can be inferred as having an upwardly directed curvature above the intraspine. In the case of our vector magnetograms, the inversions provide the magnetic parameters at a single optical depth in the atmosphere. It has been recognized that the simple inversions based on magnetic parameters independent of optical depth are consistent with a weighted mean of the optical depth dependent inversions like SIR (Westendorp Plaza et al. 1998). We can thus conjecture that our locations of positive tension are manifestations of the upwardly directed field curvatures that would be seen in SIR inversions. This conjecture must be verified with detailed SIR inversions over the entire sunspot, which will be presented in a future, detailed paper. For the present, we can only point out that the locations of positive tension in the penumbra appear to be in the regions of lower field strength and larger inclination, which are the properties of the intraspine. We can thus conclude that the lateral variation of the magnetic field, as inferred from simplistic inversions of the stokes profiles, appear to show an upwardly directed tension, which may in fact be located above the intraspine.
Likewise, the upwardly directed tension seen in light bridges could be due to a similar bending of field lines around a more horizontal field (Sankarasubramanian \& Hagenaar 2007). Finally, the positive tension seen at the edges in bipolar sections of the filamentary penumbral structures could well be a signature of the "sea-serpent" (Sainz Dalda \& Bellot Rubio 2008) where the penumbral fields dip into the photosphere due to hydrodynamic forces caused by convection.

Apart from the sign, the magnitude of the vertical tension also has some very interesting implications. As mentioned earlier, the magnitude of tension attains values up to $10^{-2}$ dynes $/ \mathrm{cm}^{3}$ in some places. This value is comparable to the force of gravity that ranges from $5 \times 10^{-3}$ dynes $/ \mathrm{cm}^{3}$ for the quiet Sun density to $10^{-2}$ dynes $/ \mathrm{cm}^{3}$ for umbral densities. This must mean that nonmagnetic forces alone will not be able to balance this tension force. The only other force that can match this force is the gradient of magnetic pressure. As an example, we can use the values of spine $(\sim 2000 \mathrm{G})$ and intraspine $(\sim 1000 \mathrm{G})$ from Borrero et al. (2008) and a scale height $\Lambda$ of $\sim 100 \mathrm{~km}$ for the vertical variation in field strength to estimate a downward magnetic pressure gradient $\left(\frac{\Delta B^{2}}{8 \pi \Lambda}\right)$ of $\sim 1.2 \times 10^{-2} \mathrm{dynes} / \mathrm{cm}^{3}$. This value is close to that of the magnetic tension obtained in our analysis. It is well known that configurations where the magnetic tension balances the magnetic pressure gradient are the socalled force-free configurations. Thus, the use of high-resolution magnetograms leads to the important result that the existence of upward magnetic tension in sunspot penumbral fine structure could be a manifestation of uncombed magnetic field in forcefree equilibrium.

Since the observations refer to values at a given optical depth, the resulting magnetogram is not at a single geometrical height. What could be the effect of this corrugation in the height levels? One can estimate that the relative amplitude of tension fluctuations $(\Delta T / T)$ will be proportional to the relative amplitude in the field variations $(\Delta B / B)$. We obtain $\Delta B$ of $\sim 100 \mathrm{G}$ by assuming a vertical gradient of magnetic field of $1 \mathrm{G} / \mathrm{km}$ in the penumbra and a corrugation amplitude of $100 \mathrm{~km}$ for the optical depth. Knowing that the penumbral fields are $\sim 1000 \mathrm{G}$, we can estimate the relative field variation as $10 \%$, which then is also the estimate for the relative variation in the tension force. The observed spatial variation in tension is much greater than this estimated artefact in tension fluctuations.

We must remember that the 180 degree ambiguity is not a problem whenever the transverse magnetic vector makes an angle of less than 90 degrees with the potential field. In all such cases, the ambiguity is properly resolved by the acuteangle method. The NOAA AR 10933 is away from the polarity inversion line and is not highly sheared (Tiwari et al. 2009b; Venkatakrishnan \& Tiwari 2009). The locations of positive tension discussed thus far all have transverse vectors that are not perpendicular to the potential fields. So they have no problem as far as the acute angle method is concerned. We do see, however, that this will be a problem for highly sheared portions of the magnetic field. We normally expect low values of magnetic tension at such highly sheared regions. But for AR 10930, there is strong magnetic tension seen in the highly sheared regions at the polarity inversion line (Fig. 3). In such cases, we need to have a careful look at the 180-degree ambiguity resolution before we can decide on the sign of magnetic tension.

However, the upward tension could indeed be a signature of the forces that drive flux emergence (Lites \& Low 1997). This conjecture is consistent with the copious emergence of flux observed on 12 December 2006 at the polarity inversion line (PIL) of the AR 10930 (Schrijver et al. 2008). In contrast, the PIL of 


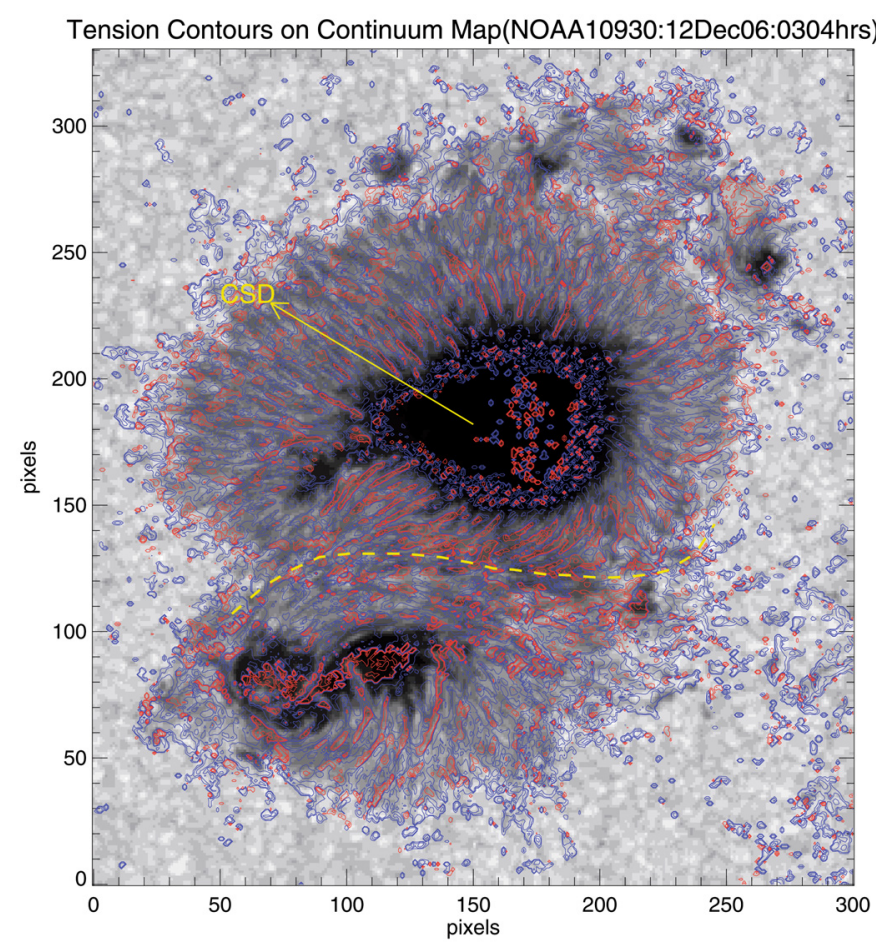

Fig. 3. Contours of tension forces overlaid on the continuum map of NOAA AR 10930 observed on 12 December 2006 at an heliocentric angle of $\theta=31^{\circ}$. Blue (red) colors show negative (positive) contours of $\pm 1.2, \pm 4, \pm 12$ millidynes $/ \mathrm{cm}^{3}$. Polarity inversion line is shown by dashed yellow line. An arrow points towards the center of the solar disk (CSD).

AR 10930 on 14 December 2006 in Fig. A.3 (online) shows a reduced value of the tension corresponding to a time of negligible flux emergence. Thus, the study of magnetic tension in various types of sunspot fine structures promises to yield new and exciting information on the equilibrium and dynamics of these structures.

Acknowledgements. We thank Professors E. N. Parker and S. M. Chitre for reading the manuscript, and Professors B. C. Low and R. Moore for their valuable suggestions for improving the manuscript. We thank an anonymous referee for his/her useful suggestions and comments to improve the manuscript. Hinode is a Japanese mission developed and launched by ISAS/JAXA, with NAOJ as domestic partner and NASA and STFC (UK) as international partners. It is operated by these agencies in co-operation with ESA and NSC (Norway).

\section{References}

Beck, C. 2008, A\&A, 480, 825

Borrero, J. M., Lites, B. W., \& Solanki, S. K. 2008, A\&A, 481, L13

Chitre, S. M. 1963, MNRAS, 126, 431

Collados, M., Martinez Pillet, V., Ruiz Cobo, B., del Toro Iniesta, J. C., \& Vazquez, M. 1994, A\&A, 291, 622

Cuperman, S., Li, J., \& Semel, M. 1992, A\&A, 265, 296

del Toro Iniesta, J. C. 2003, Astron. Nachr., 324, 383

Del Toro Iniesta, J. C., \& Ruiz Cobo, B. 1996, Sol. Phys., 164, 169

Gosain, S., Tiwari, S. K., \& Venkatakrishnan, P. 2010, ApJ, submitted

Hagyard, M. J., Cumings, N. P., West, E. A., \& Smith, J. E. 1982, Sol. Phys., 80, 33

Harvey, J. W. 1969, Ph.D. Thesis, University of Colorado, Boulder

Ichimoto, K., Lites, B., Elmore, D., et al. 2008, Sol. Phys., 249, 233

Kosugi, T., Matsuzaki, K., Sakao, T., et al. 2007, Sol. Phys., 243, 3

Landolfi, M., \& Landi Degl'Innocenti, E. 1982, Sol. Phys., 78, 355

Lites, B. W., \& Low, B. C. 1997, Sol. Phys., 174, 91

Lites, B. W., Elmore, D. F., Seagraves, P., \& Skumanich, A. P. 1993, ApJ, 418, 928

Meyer, F., Schmidt, H. U., \& Weiss, N. O. 1977, MNRAS, 179, 741

Parker, E. N. 1979, Cosmical magnetic fields: Their origin and their activity (Oxford: Clarendon Press; New York: Oxford University Press)

Rachkowsky, D. N. 1967, Izv. Krymsk. Astrofiz. Obs., 37, 56

Ruiz Cobo, B., \& del Toro Iniesta, J. C. 1992, ApJ, 398, 375

Ruiz Cobo, B., \& del Toro Iniesta, J. C. 1994, A\&A, 283, 129

Sainz Dalda, A., \& Bellot Rubio, L. R. 2008, A\&A, 481, L21

Sakurai, T., Makita, M., \& Shibasaki, K. 1985, MPA Rep., 212, 312

Sankarasubramanian, K., \& Hagenaar, H. 2007, Bull. Astron. Soc. India, 35, 427

Schrijver, C. J., De Rosa, M. L., Metcalf, T., et al. 2008, ApJ, 675, 1637

Shimizu, T., Nagata, S., Tsuneta, S., et al. 2008, Sol. Phys., 249, 221

Skumanich, A., \& Lites, B. W. 1987, ApJ, 322, 473

Sobotka, M. 1989, Sol. Phys., 124, 37

Sobotka, M., Brandt, P. N., \& Simon, G. W. 1997a, A\&A, 328, 682

Sobotka, M., Brandt, P. N., \& Simon, G. W. 1997b, A\&A, 328, 689

Solanki, S. K., \& Montavon, C. A. P. 1993, A\&A, 275, 283

Spruit, H. C., \& Scharmer, G. B. 2006, A\&A, 447, 343

Suematsu, Y., Tsuneta, S., Ichimoto, K., et al. 2008, Sol. Phys., 249, 197

Tiwari, S. K. 2009, Ph.D. Thesis, Udaipur Solar Observatory/Physical Research Laboratory, Mohanlal Sukhadia University, Udaipur

Tiwari, S. K., Venkatakrishnan, P., Gosain, S., \& Joshi, J. 2009a, ApJ, 700, 199

Tiwari, S. K., Venkatakrishnan, P., \& Sankarasubramanian, K. 2009b, ApJ, 702, L133

Tsuneta, S., Ichimoto, K., Katsukawa, Y., et al. 2008, Sol. Phys., 249, 167

Unno, W. 1956, PASJ, 8, 108

Venkatakrishnan, P. 1990a, in Basic Plasma Processes on the Sun, ed. E. R. Priest, \& V. Krishan, IAU Symp., 142, 323

Venkatakrishnan, P. 1990b, Sol. Phys., 128, 371

Venkatakrishnan, P., \& Tiwari, S. K. 2009, ApJ, 706, L114

Venkatakrishnan, P., Narayanan, R. S., \& Prasad, N. D. N. 1993, Sol. Phys., 144, 315

Westendorp Plaza, C., del Toro Iniesta, J. C., Ruiz Cobo, B., et al. 1998, ApJ, 494, 453

Westendorp Plaza, C., del Toro Iniesta, J. C., Ruiz Cobo, B., \& Martínez Pillet, V. 2001a, ApJ, 547, 1148

Westendorp Plaza, C., del Toro Iniesta, J. C., Ruiz Cobo, B., et al. 2001b, ApJ, 547,1130

Page 5 is available in the electronic edition of the journal at http://www . aanda.org 
P. Venkatakrishnan and Sanjiv Kumar Tiwari: Magnetic tension of sunspot fine structures

\section{Appendix A: Additional figures}

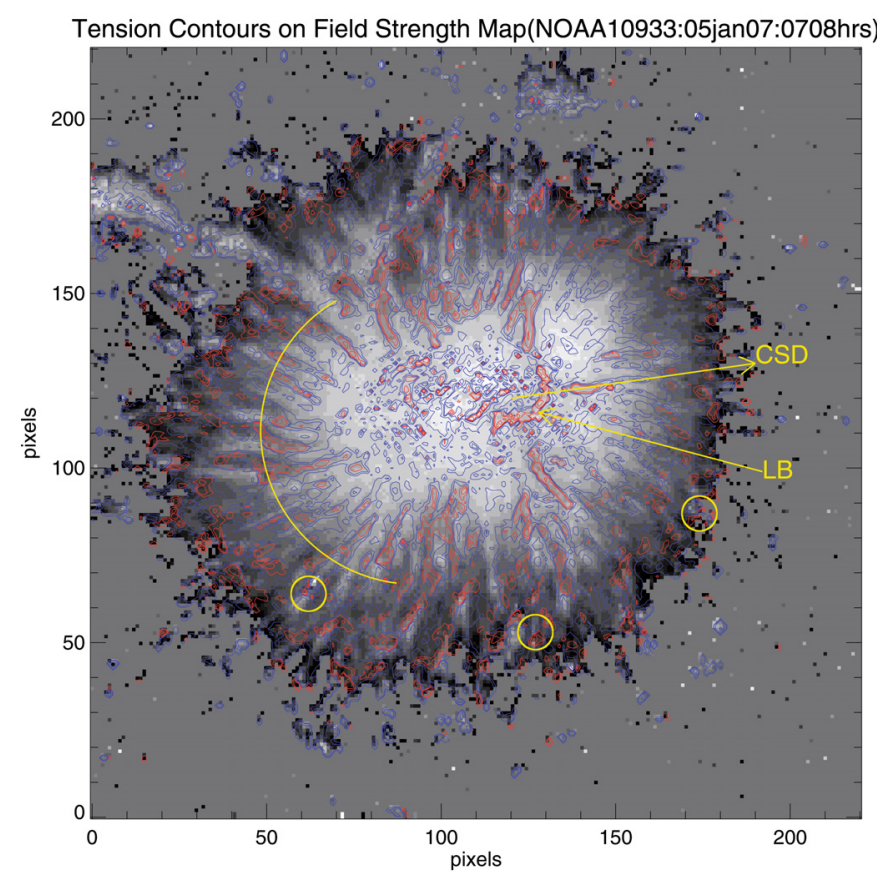

Fig. A.1. Same as that of Fig. 1, except that the continuum image is replaced by the field strength map of NOAA AR 10933. Contour levels are $\pm 1.2, \pm 4, \pm 12$ millidynes $/ \mathrm{cm}^{3}$.

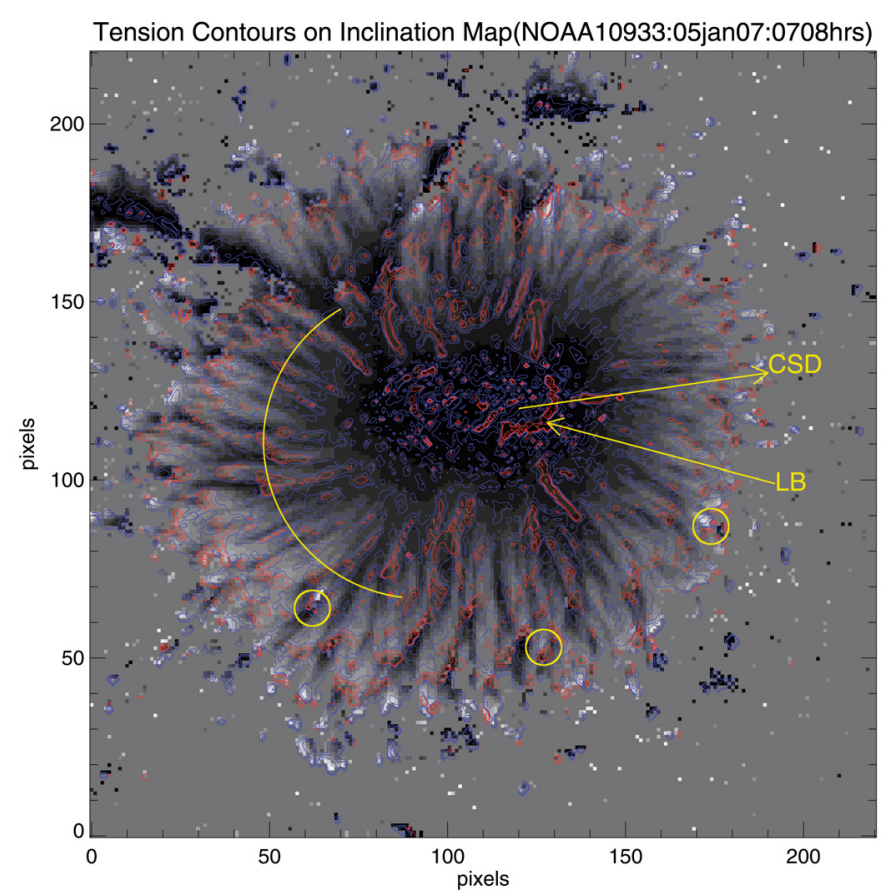

Fig. A.2. Same as that of Fig. 1, except that the continuum image is replaced by the field inclination map of NOAA AR 10933.

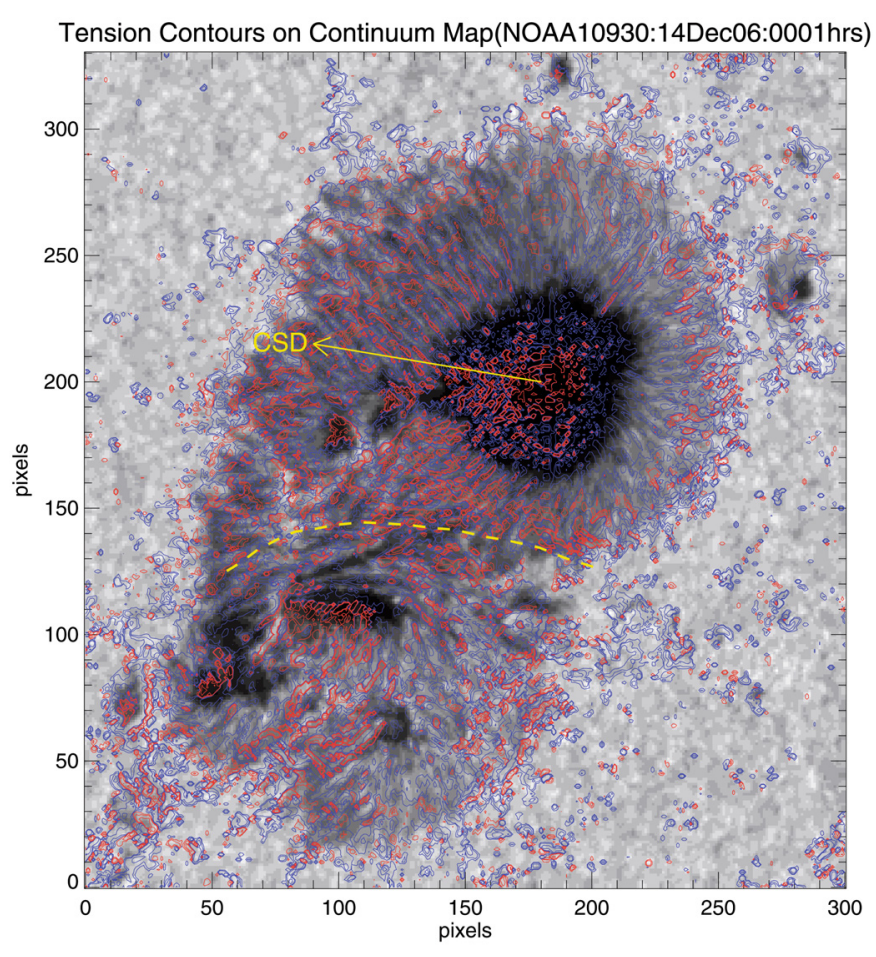

Fig. A.3. Same as that of Fig. 3, except that this sunspot is observed on 14 December 2006 at a heliocentric angle of $\theta=8^{\circ}$. 